\title{
Comparison of Punica granatum, Terminalia chebula, and Vitis vinifera Seed Extracts used as Mouthrinse on Salivary Streptococcus mutans Levels in Children
}

\author{
Pooja Mishra ${ }^{1}$, Nikhil Marwah ${ }^{2}$, Neha Agarwal $^{3}$, Yogita Chaturvedi ${ }^{4}$, Thejavinuo Suohu ${ }^{5}$
}

\begin{abstract}
Aim: The present study was conducted to compare the efficacy of all Punica granatum, Terminalia chebula, and Vitis vinifera on salivary Streptococcus mutans levels in children and also to evaluate their substantivity at an interval of 15 days that is at day 1, days 16 , and 31 .

Materials and methods: This study was designed for a randomized clinical double-blinded study where 80 children of 8-15 years of age were living in a residential premise. Subjects were randomly divided into 4 groups of 20 each to whom mouthrinses were given. The criteria for assessing the efficacy was done by collecting the saliva sample for $\mathrm{pH}$, buffering capacity, plaque index, and Streptococcus mutans microbiologic assay. These values were assessed at the baseline, days 16, and 31. Children were asked to discontinue mouthrinse from days 16 to 31 . The supervisor was trained to administer the mouthrinses properly.

Results: The data were coded and analysis was done using the SPSS version 20. The level of significance was set at $p<0.05$. The $\mathrm{pH}$ and buffering capacity showed that values were almost the same among all four groups at various time intervals which showed statistically nonsignificant results. Punica granatum showed a maximum reduction in S. mutans count followed by T. chebula and $V$. vinifera, although they were statistically nonsignificant. The Vitis vinifera group had successfully reduced more plaque score at day $16(0.04)$ followed by T. chebula (0.09) and P. granatum (0.12).

Conclusion: This in vivo study implied that $V$. vinifera had shown the lowest plaque reduction owing to its antioxidant and phytochemical properties. And P. granatum showed the maximum substantivity.

Clinical significance: Mouthrinses helped in reducing plaque deposition, caries activity, and helped in oral hygiene maintenance. Hereby, we can conclude that nutraceutical mouthrinses are safe in children and produced superior results than the chemical mouthrinses.

Keywords: Buffering capacity, Mouthrinse, Nutraceutical, pH, Saliva, Streptococcus mutans.

The Journal of Contemporary Dental Practice (2019): 10.5005/jp-journals-10024-2617
\end{abstract}

\section{INTRODUCTION}

Dental caries can aptly be termed as scourge of modern civilization and no nation or community has escaped the ill effects of this malady. Nowadays, the global community is looking for sustainable solutions to the age-related onset of fatal diseases across different healthcare systems. Accordingly, a question is raised, if Ayurveda, the world's most comprehensive, personalized, holistic healthcare system, can provide answers to intercept the disease process.

Presently, there has been a prodigious interest worldwide in nutraceuticals, which are known to play a pivotal role in health management. This is due to their presumed safety and potential nutritional and therapeutic benefit for mankind. ${ }^{1}$ Many naturally occurring compounds found in dietary and medicinal plants and fruits have been shown to possess antimicrobial activities ${ }^{7}$ which definitely has beneficial effects on general health as well as on oral health of the mankind.

Cariogenic plaque hasthe property of producing acid from available fermentable substrates by definitive plaque bacteria resulting in the destruction of enamel during the formation of the carious lesion. An oral mouthwash based on the specific plaque hypothesis is an effective chemotherapeutic agent which possesses an effective and immediate home care remedy to enhance oral hygiene and prevent dental caries by objecting the cariogenic bacteria.

Chlorhexidine has been extensively used in almost all medicinal branches. The antimicrobial property of chlorhexidine
${ }^{1-5}$ Department of Pedodontics, Mahatma Gandhi Dental College, Jaipur, Rajasthan, India

Corresponding Author: Pooja Mishra, Department of Pedodontics, Mahatma Gandhi Dental College, Jaipur, Rajasthan, India, Phone: +91 9868786574, e-mail: poojamishra002@gmail.com

How to cite this article: Mishra P, Marwah N, et al. Comparison of Punica granatum, Terminalia chebula, and Vitis vinifera Seed Extracts used as Mouthrinse on Salivary Streptococcus mutans Levels in Children. J Contemp Dent Pract 2019;20(8):920-927.

Source of support: Nil

Conflict of interest: None

was attributed to its di-cationic structure. Both Gram-positive and Gram-negative bacterial strains are susceptible to chlorhexidine. Following single rinse with chlorhexidine, saliva itself exhibits antibacterial sustainability for extended hours and obstructs the salivary bacterial counts for more than 12 hours. As a mouthrinse, the di-cationic nature of chlorhexidine depicts a wide range of side effects, causing extrinsic tooth staining, altered taste, and so on. Because of the mentioned side effects, tremendous research is in progress for an antibacterial agent which is equivalent to chlorhexidine but with less or negligible side effects.

In recent times, there has been a remarkable shift toward natural cures because of the pronounced cumulative and irreversible reactions of chemicals. Antimicrobial property of

() The Author(s). 2019 Open Access This article is distributed under the terms of the Creative Commons Attribution 4.0 International License (https://creativecommons. org/licenses/by-nc/4.0/), which permits unrestricted use, distribution, and non-commercial reproduction in any medium, provided you give appropriate credit to the original author(s) and the source, provide a link to the Creative Commons license, and indicate if changes were made. The Creative Commons Public Domain Dedication waiver (http://creativecommons.org/publicdomain/zero/1.0/) applies to the data made available in this article, unless otherwise stated. 
natural food due to increased phenolic content has been studied and their action against pathogenic microbes antiquated intensive investigation to characterize and develop a healthy product which can be used for mankind.

Pomegranate (Punica granatum), a prominent member of the Punicaceae family, is a shrub found in Asia and is outspread in the Mediterranean region. From biblical times, this fruit has been used widely and depicts therapeutic properties. ${ }^{2}$ Pomegranate fruit (Fig. 1A) is one of the delicious edible natural products which can be consumed as fresh, fruit juice and as an ingredient in the preparation of jellies, jams, and also for flavoring and coloring beverage products. ${ }^{3}$ The presence of high amounts of bioactive compounds, such as phenolic acids, flavonoids, and tannins in pomegranate fruits, assures them rich nutritive value. ${ }^{4}$ Literature suggests that extracts of pomegranate play an important role in preventing bacteria and thereby reducing the risk of plaque, gingivitis, and periodontal disease. Authors have inferred that the ellagitannin and punicalaginare mainly responsible for the antibacterial activity of pomegranate extract rinses. Thereby, the use of pomegranate extracts in oral health products such as toothpastes and mouthwashes could be a sustainable option. ${ }^{5}$

The plant T. chebula is a medicinal plant known as "harada" in Hindi and "kadukka" in Tamil. It is popularly known as black or chebulicmyrobalan. This plant is a species of Terminalia which is
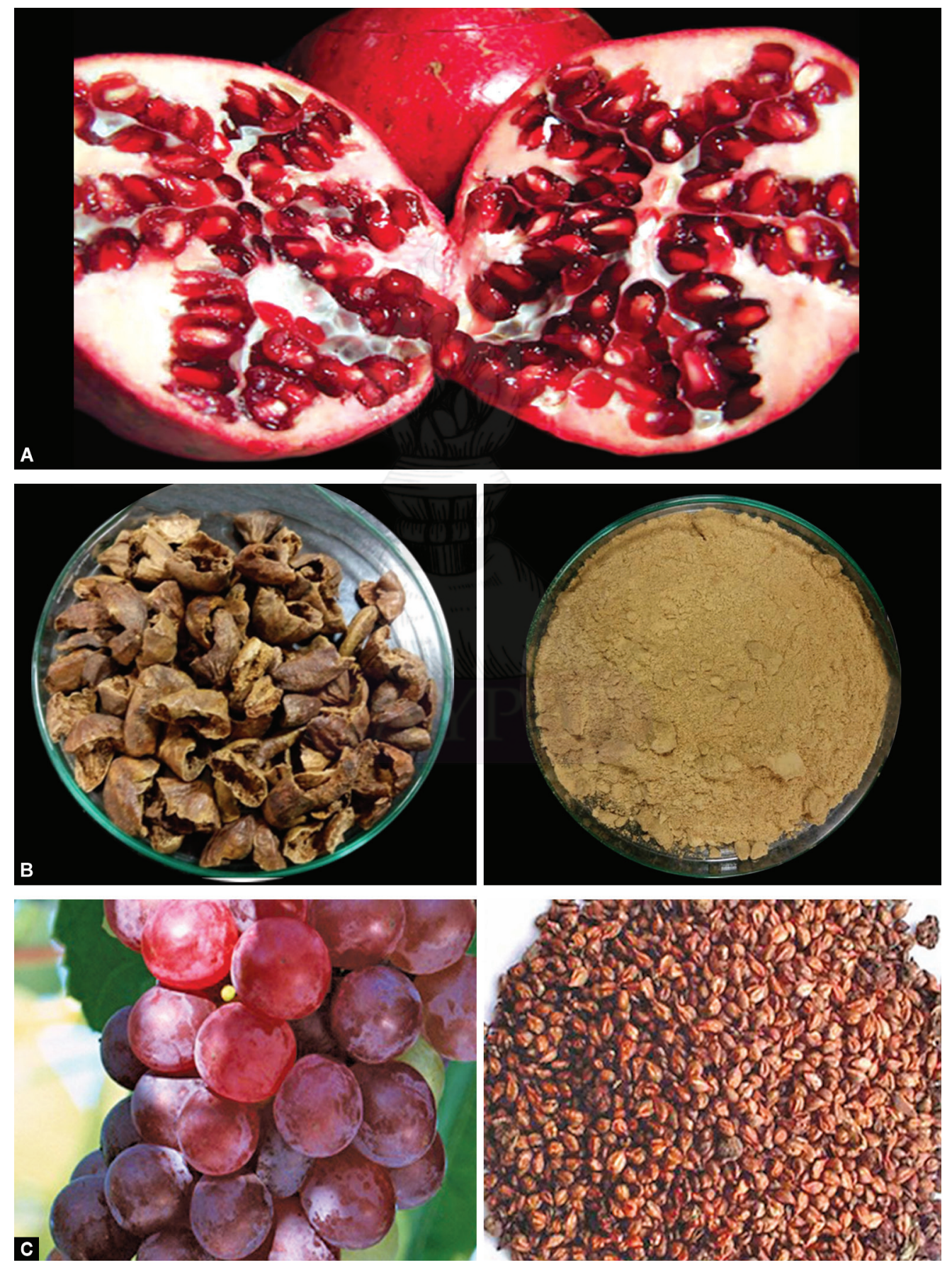

Figs 1 A to C: (A) The fruit of Punica granatum; (B) The dry form and powdered form of Terminalia chebula; (C) The fruit of Vitis vinifera and its seeds 
native to India and Nepal. Terminalia chebula belongs to the family of Combretaceae which possesses anticarogenic properties in addition to antidiabetic, anti-inflammatory, and antioxidant effects. The ripe fruits of this fruit (Fig. 1B) prevent dental cariesand gingivitis, and studies conducted in the past showed that mouthwashes containing T. chebula have antimicrobial effects against oral bacteria and possess a long history of use. ${ }^{6}$ This fruit iscomposed of $32-34 \%$ tannins and other polyphenols like galloyl glucose, punicalagin, terflavin A, and malinic acid. ${ }^{8-10}$ Terminaliachebula depicted anti-bacterial activity against Gram-positive and Gram-negative pathogenic bacteria, anti-fungal, and anti-viral properties. ${ }^{11}$ Traditionally, the dried ripe fruits are beneficial in the treatment of asthma, sore throat, vomiting, hiccup, and diarrhea. ${ }^{12}$

Vitis vinifera is a member of the Vitaceae family, native to the Mediterranean region, central Europe, and southwest Asia and cultivated today in all the temperate regions of the world. ${ }^{13}$ Grape seeds are known for its rich sources of polyphenolic compounds, mainly catechin and epicatechin, gallic acid, and procyanidin ${ }^{14}$ which show both antioxidant andantimicrobial properties. ${ }^{15}$ Phenolic contents in grape seeds are partially hydrophobic and are considered to interact with the bacterial cell wall and lipopolysaccharide interfaces by decreasing the membrane stability. The phenolic content in grape seed extract (GSE) has been directly correlated to the antibacterial properties. ${ }^{15}$ Vitis vinifera seeds (Fig. 1C) are considered rich sources of polyphenolic compounds mainly procyanidins, proanthocyanidins, anthocyanins, flavonols, and phenolic acids. GSE constitutes high proanthocyanidins culminating in positive remineralization and/or demineralization process of artificial root caries lesions done in vitro, suggesting its possibility as a promising natural agent for noninvasive root caries therapy. ${ }^{16}$

However, only in vitro studies have been reported on $V$. vinifera extracts, and no in vivo studies have been done to check its efficacy on S. mutans count. Owing to disadvantages of chlorhexidine, and the known popularity of herbal and Ayurvedicformulations including their potential positive effects, it was decided to compare the three herbal mouthwashes in vivo and evaluate their comparison with the gold standard chlorhexidine, as the control group. The intention of the study was to evaluate the change in the salivary $\mathrm{pH}$, buffering capacity, and microbial count of the saliva after using extracts of $P$. granatum, $T$. chebula, and $V$. vinifera and $0.2 \%$ of chlorhexidine as a mouthwash in children.

\section{Materials and Methods}

The purpose of the study was to check the change in the salivary $\mathrm{pH}$, buffering capacity, and reduction in the microbial count of the saliva before and after rinsing with the extract of $P$. granatum, T. chebula, and $V$. vinifera and $0.2 \%$ of chlorhexidine as a mouthwash in children. An ethical committee approval was obtained from the Ethical Committee, Mahatma Gandhi Dental College and Hospital prior to the onset of the study. The nature of the study was fully explained and verbal consent from children and signed consent from caretakers and teachers were obtained.

The study was designed for a randomized clinical doubleblinded study where 80 children of 8-15 years of age living in one residential premise were selected for this study. They were subjected to clinical examination and a sampling frame was prepared of those who were at an increased risk of dental caries that is with decayed missing filled teeth (DMFT)/decayed, missing and filled teeth $(\mathrm{dmft})>4$ and no history of an orthodontic appliance and no medical history were the inclusion criteria. Children were
Table 1: Method of preparing nutraceutical mouthwash

\begin{tabular}{ll}
\hline Pippermint oil 0.5\% & $50 \mathrm{~mL}$ \\
Alum 1\% & $100 \mathrm{~g}$ \\
Copper sulfate $0.5 \%$ & $50 \mathrm{~g}$ \\
Tulsi extract (18:1) & $100 \mathrm{~g}$ \\
Sodium benzoid & $20 \mathrm{~g}$ \\
R.O. water & Rest \\
Extracts of different herbs were added separately & \\
\hline
\end{tabular}

randomly allocated into the study groups and the study model wasdecided to be a double-blinded study.

The children of each group were then instructed to rinse their mouth with $10 \mathrm{~mL}$ of respective mouth rinse once daily after dinner for 15 days. The children were asked regarding any adverse or unusual reactions pertaining to the use of various mouth rinses upon the next visit.

The mouthwashes were prepared under the guidance of Dr Abhyudaya Sharma Production Manager, Sri Navjeewan Rasayanshala, Jaipur. The basic formula used for the preparation and extracts as per need, palatability, and color (Table 1).

Fruit extracts from $P$. granatum and $T$. chebula were collected and cut into small pieces and then blended and powdered. Grape seed extracts were dissolved in sterile distilled water with gentle heating and various dilutions were made by doubling the dilutions and was kept in separate beaker. The fine powder of alum was mixed in peppermint oil. Copper sulfate was dissolved in demineralized (DM) water separately. Both the above groups were mixed well (alum andcopper sulfate) and werefiltered through a filter press and the solution was allowed to cool off. Later, dried and powdered extract of $P$. granatum, $T$. chebula, and $V$. vinifera were added to the above solution. This was mixed using a stirrer until a uniform color was obtained. The solution was left for overnight. On the next following day, the solution was filled in a bottle of $150 \mathrm{~mL}$ and packed. The shelf life of 18 months was obtained (Fig. 2).

Each individual was made to sit and relax comfortably and the pooled unstimulated whole saliva was collected by spitting in the collecting sterile tubes. ${ }^{17}$ The method approached for collection of saliva was given by Mandel ${ }^{17}$ as it was found convenient and easy to use. No transport media was used, and samples were cultured within 30 minutes of collection of saliva samples. The samples were then subjected to microbiology tests.

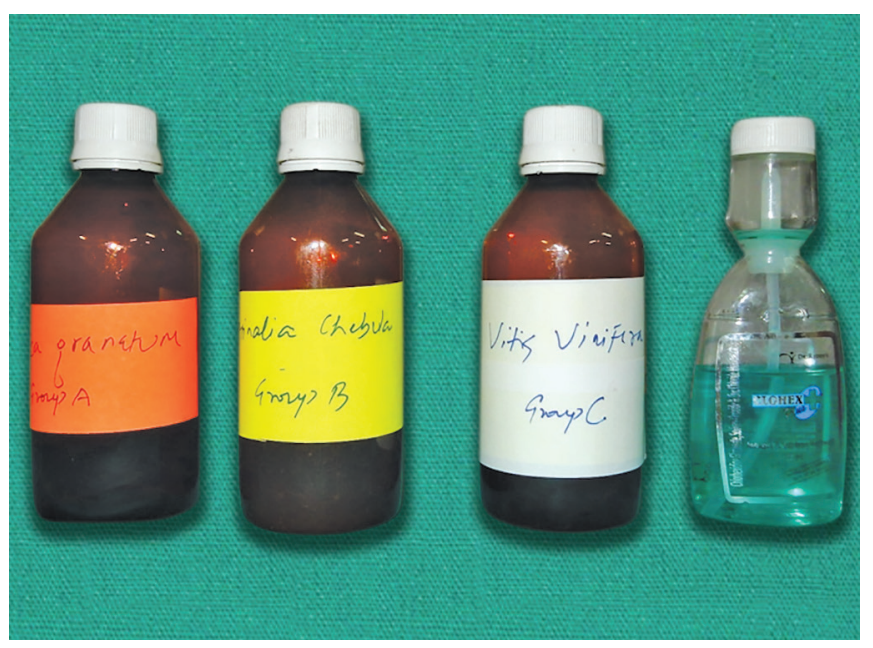

Fig. 2: Mouthrinses dispersed to children in study 


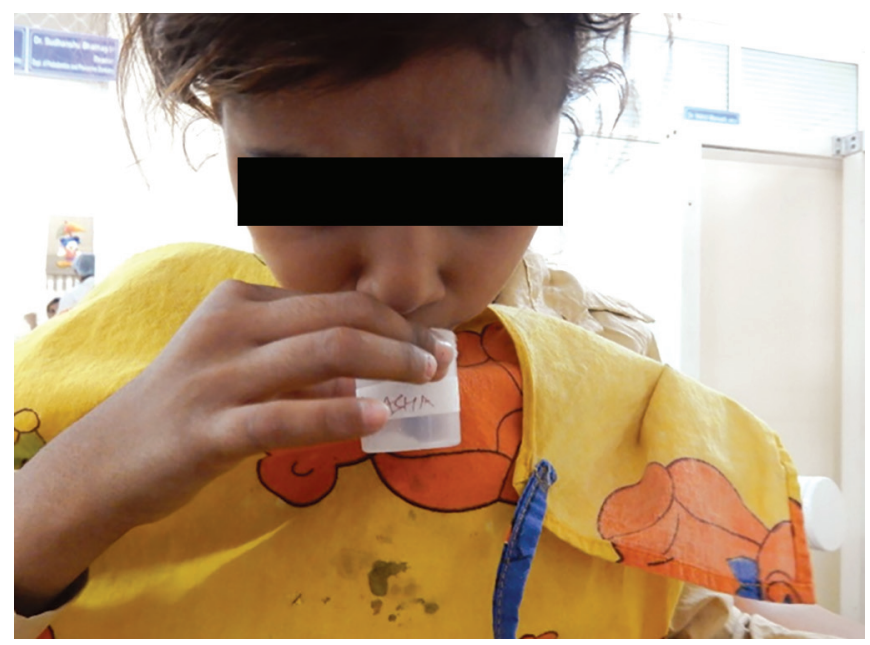

Fig. 3: Method of collection of saliva

Mitis Salivarius Bacitracin Agar media was used for the isolation of S. mutans. Baseline salivary samples were collected after oral prophylaxis (Fig. 3). After the calculus was removed, the alpha plaque disclosing agent was applied with cotton buds and a visible plaque was removed. Plaque scores were then recorded according to "Turesky-Gilmore-Glickman modification of the Quigley-Hein Plaque Index"with the help of a volunteer. Saliva samples were collected from subjects and were estimated for $\mathrm{pH}$ and buffering capacity. The pH was checked by the GC Salivary Kit (Fig. 4) and the buffering capacity was measured by the titration method (Fig. 5).

On the 16th day, unstimulated saliva was collected from the subjects of all fourgroups, inoculated onto the Mitis-Salivarius bacitracin (MSB) agar plate and colony counts were obtained. The plaque index was also recorded at this juncture. For the next 15 days, the subjects in all groups discontinued the mouthwash and maintained regular tooth brushing twice daily. And plaque score, $\mathrm{pH}$, buffering capacity, and the salivary S. mutans colony-forming units were evaluated again on day 31 .

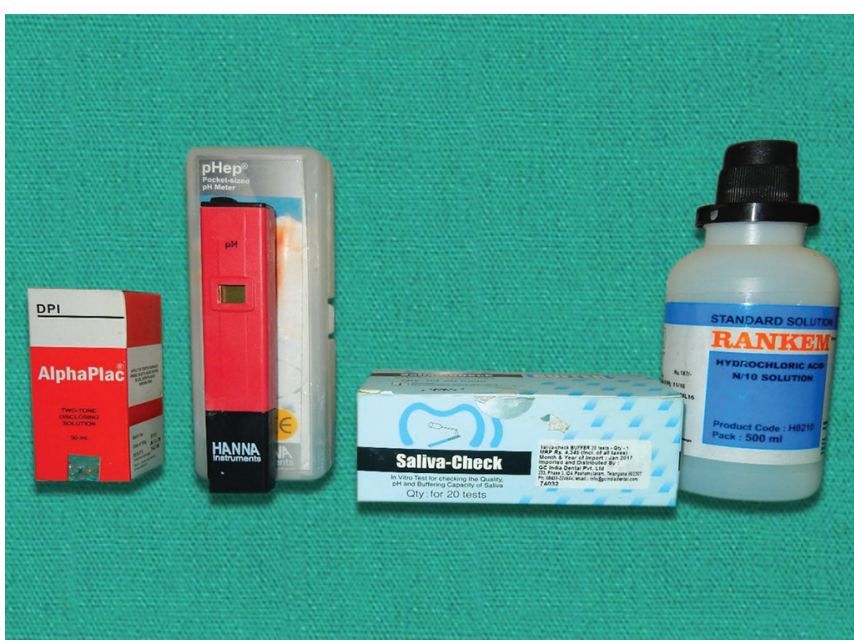

Fig. 4: Materials used for determining plaque index (DPI Alpha plaq), $\mathrm{pH}$ (handheld pH meter, GC Saliva check), and buffering capacity (hydrochloric acid $\mathrm{N} / 10$ solution)

Colonies observed as round or spherical, raised, convex, dark in color, ranging from a pin point to pin head size with a rough surface were identified as $S$. mutans colonies in $T$. chebula, $P$. granatum, and $V$. vinifera and the control group at the baseline, days 16 , and 31, respectively (Fig. 6). Identification of S. mutans was confirmed by Gram staining of the smear and microscopy followed by biochemical tests like the catalase test. The colony unit of each plate was recorded and the mean colony forming units $(\mathrm{CFU} / \mathrm{mL})$ was determined after multiplying the colony count of each plate with its respective dilution factor.

The result was then sent for statistical analysis.

\section{Result}

The data werecoded and entered into the Microsoft Excel spreadsheet. The analysis was done using the SPSS version 20
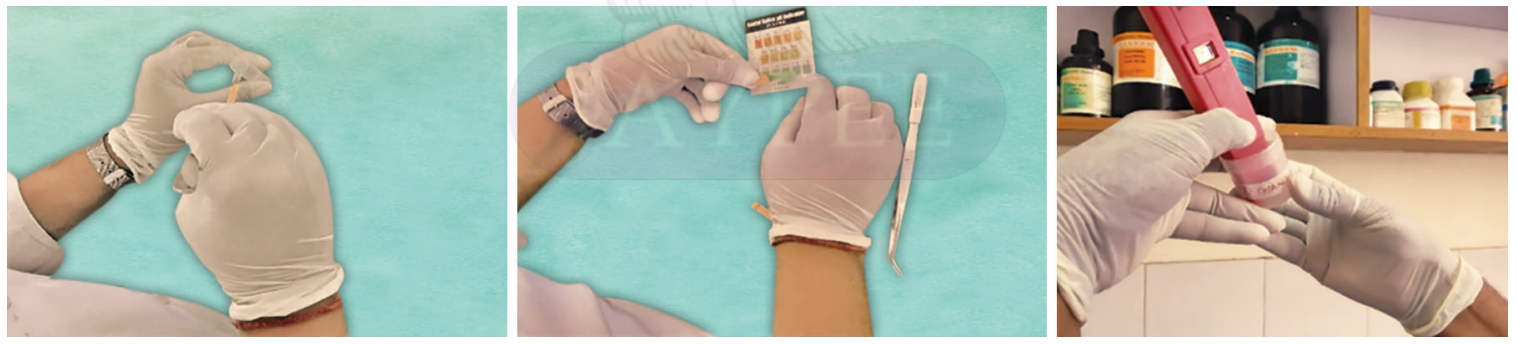

Fig. 5: Determination of $\mathrm{pH}$ and buffering capacity
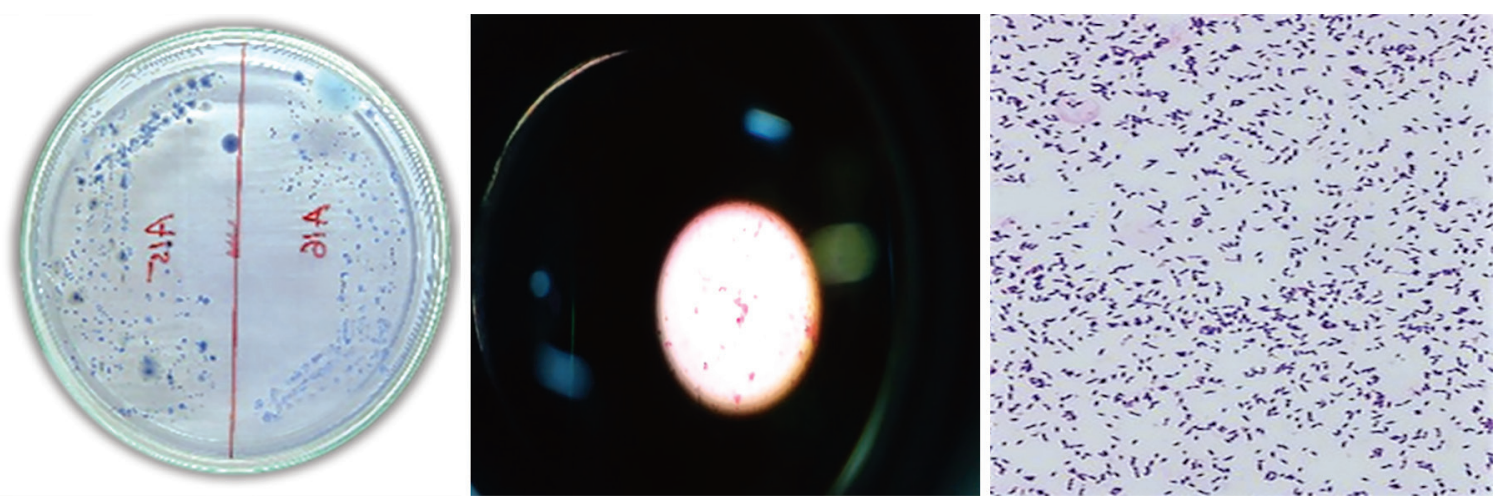

Fig. 6: Streptococcus mutans colony in Gram staining under 40× magnification (microscopic view) 
(IBM SPSS Statistics Inc., Chicago, Illinois, USA) Windows software program. The variables were assessed for normality using the Kolmogorov-Smirnov test. Descriptive statistics included computation of percentages, means, and standard deviations. The analysis of variance (ANOVA) test wasused for a comparison of all clinical indicators. The Chi-square test used for qualitative data whenever two or more than two groups were used to compare. The level of significance was set at $p \leq 0.05$.

The comparison of plaque index score among groups using the ANOVA test revealed that the control group had a mean score of plaque score at days $16(0.28)$ and 31 (1.09) which showed statistically significant results. In $P$. granatum, the plaque score showed the plaque value at day 1 (1.59) which decreased at day $16(0.12)$, but then after we have noticed that on day 31, there were slightly increased scores (0.69), which is lesser than all groups pointing to be more substantive. If $V$. vinifera is compared at day 1 , days 16 , and 30 , it presents the highest value of the plaque score at day 1 (1.99) which decreased at day $16(0.04)$, but then on the 31st day, the score increased significantly (1.02) which makes it less substantive than $P$. granatum. Terminalia chebula represents the highest value of the plaque score at day 1 (2.16) which decreased

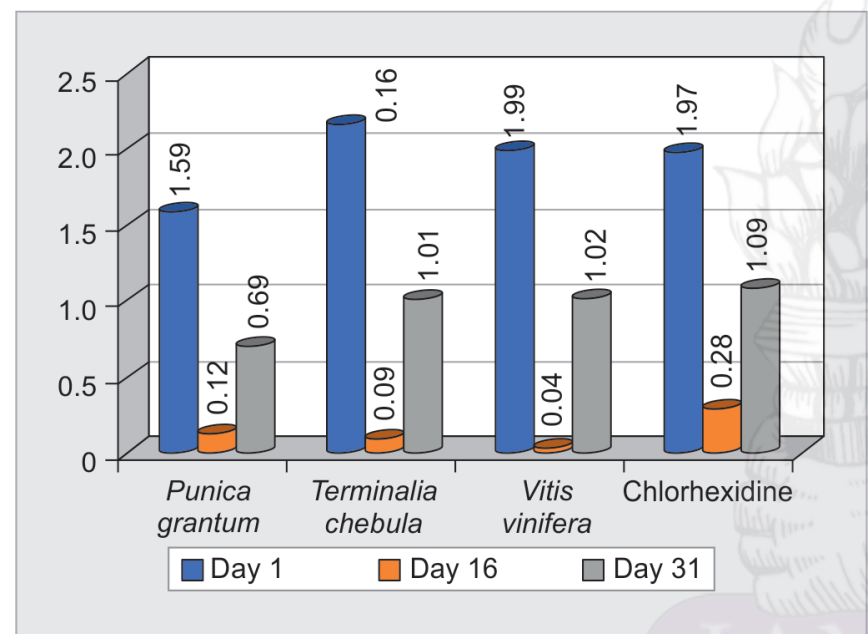

Fig. 7: Mean of the plaque index scores among Punica granatum, Terminalia chebula, Vitis vinifera, and chlorhexidine

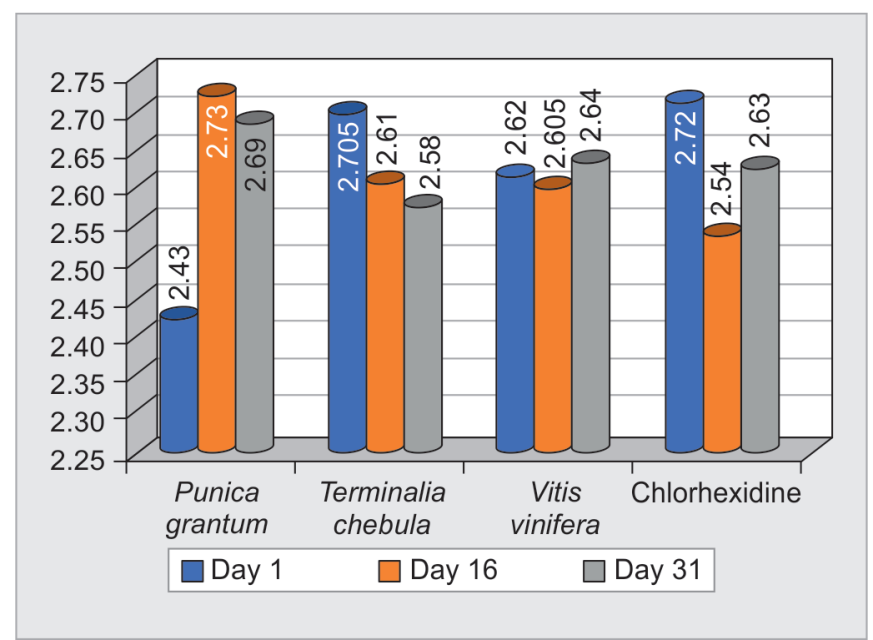

Fig. 9: Mean of buffering capacity among Punica granatum, Terminalia chebula, Vitis vinifera, and chlorhexidine at day 16 (0.09) but then an increased score was noticed on day 31 (1.01) which showed statistically significant results (Fig. 7). The $\mathrm{pH}$ value showed a gradually decreased score from day 1, days 16 , and 31 in all groups (Fig. 8). The mean of buffering capacity was almost the same among all four groups at various time intervals which showed a statistically nonsignificant result. Punica granatum at day 1 (2.43), days 16 (2.73), and 31 (2.69) shows that over the period, it was successful in maintaining buffering capacity. Similarly, T. chebula and Vitis vinifera had also managed to maintain the same as depicted (Fig. 9). Punica granatum showed a maximum reduction in S. mutans count followed by $T$. chebula and $V$. vinifera. Although the comparison of $S$. mutans colony count among groups presented that the mean value of $S$. mutans count were almost the same among all four groups at various time intervals which showed statistically nonsignificant results (Fig. 10).

\section{Discussion}

Dental caries has become one of the major oral health issues these days. Caries affects all age groups crossing barriers of ethnicity, region, and language. Nowadays, a trend for medicinal remedy has shifted from allopathic to nutraceutical products which possess

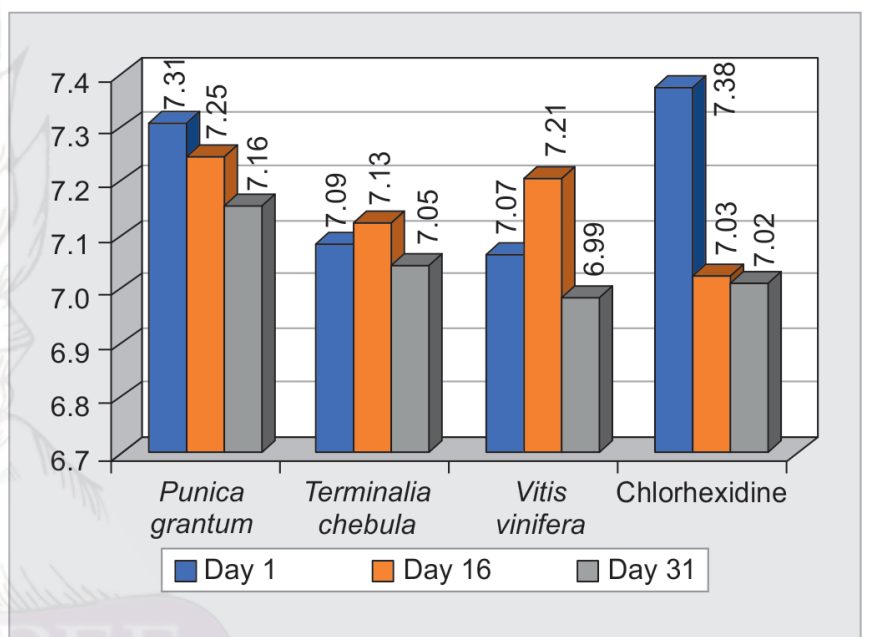

Fig. 8: Mean of $\mathrm{pH}$ value among Punica granatum, Terminalia chebula, Vitis vinifera, and chlorhexidine

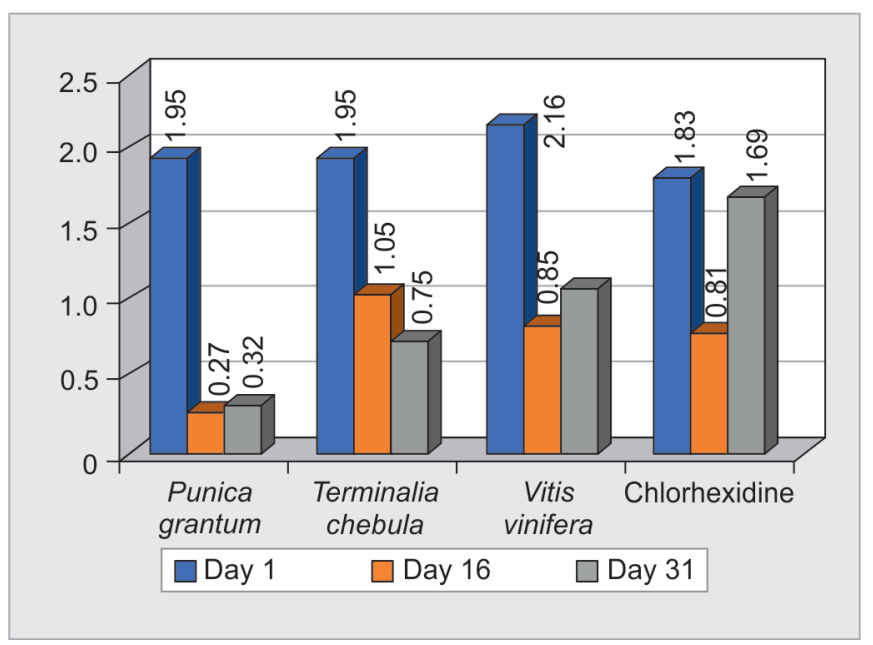

Fig. 10: Mean of Streptococcus mutans colony value among Punica granatum, Terminalia chebula, Vitis vinifera, and chlorhexidine 
fewerside effects. The plaque control agents used in this study havedetermined both efficacy and substantivity of nutraceutical mouthwashes.

This silent outbreak of oral diseases disproportionately affects children irrespective of their socioeconomic status. The age group used in our study belongs to 8-15 years old. This phase sees the transition from the primary to the mixed dentition in children. Children of this age group have a better understanding of oral hygiene and are well versed with their usage. The similar age group was chosen in a study conducted by Nayak et al., ${ }^{18}$ Anup et al., ${ }^{19}$ and Bansal et al. ${ }^{20}$

The plaque index used in this study was "Turesky-GilmoreGlickman modification of the Quigley-Hein Plaque Index". This is recognized as a reliable index for measuring plaque, using an estimate of the area of the tooth covered by plaque. ${ }^{21}$ Similar studies which have used this index were done by Gupta et al. $^{22}$ and Anup et al. ${ }^{19}$

The $\mathrm{pH}$ was measured by the chair side saliva check kit (GC India) and the buffering capacity was determined by diluting the saliva with hydrochloric acid N/10 solution and measuring the $\mathrm{pH}$ using a hand held meter. For $\mathrm{pH}$, unstimulated saliva was taken as described in the kit. It is known that resting saliva $\mathrm{pH}$ plays a prime role in caries initiation. Similar studies have used unstimulated saliva for $\mathrm{pHs}$ like Carounanidy et al., ${ }^{23}$ Nayak et al., ${ }^{18}$ and Vidhya et al. ${ }^{24}$

Principle identification of Mutans streptococci is often made by the defined morphology of its colonies on sucrose-containing culture media. In our study, culture growth was done on MSB agar medium which showed a granular surface like ground glass. They appeared tiny, hard, transparent, and adhesive to the medium and have the classical look of frosted glass. In addition to the recognition of Mutans streptococci from their culture agar media, Gram staining, distinctive cell shape on light microscopy and biochemical test were also done.

A rekindled interest in the medicinal properties of alternative natural products has gained popularity due to increasing problems of resistance to synthetic antimicrobials. ${ }^{25}$ The herbal mouthwashes dispensed to young children in our study were T. chebula, $P$. granatum, and V. Vinifera seed extracts with chlorhexidine being the control group.

Punica granatum contains widest classes of tannins and flavonoids because of which it indicates pharmacological potential depicting antioxidant activity by indirect inhibition of inflammatory markers such as tumor necrosis factor alpha. ${ }^{26}$ Pomegranates are rich in catechins which show high antioxidant activity. They play an important role as an inflammatory and antioxidant. The outer cover of pomegranate contains anti-inflammatory, antimutagenic, and antifungal properties. ${ }^{27}$ The excellent nutraceutical properties of $P$. granatum led us to formulate its mouthwash and use it in our study.

Terminalia chebula, or Harada, occurs naturally from the subHimalayan region of Nepal and northern India. Because of its extraordinary power of healing, this fruit has been termed as "King of Medicine." The medicinal activities are due to increasednumber of different types of phytoconstituents. ${ }^{28}$ The fruits are rich in tanninsmainly of pyrogallol type ${ }^{29}$ and researchershave found various different components of hydrolyzabletannins (gallic acid, chebulagic acid, punicalagin, chebulanin, corilagin, neochebulinic acid, ellagic acid, and chebulinic acid) from T. chebula. ${ }^{30}$ They alsoinclude phenolics such as chebulinic acid, ellagic acid, and anthraquinones. Punicalin, punicalagin, terflavins $B, C$, and D are found in leaves which arehelpful in opthalmia, dental caries, bleeding gums, and ulcered oral cavity. ${ }^{28}$ The powder of T. chebula plays the role of astringent in dentifrices in cases of loose gums, bleeding, and ulceration. Seeing its medicinal and antioxidative effects, $T$. chebula was chosen as the second comparative group.

Vitis vinifera are counted in the most commonly consumed fruits worldwide. The most important phenolics extracted from grape seeds are catechins (catechin, epicatechin, and procyanidins) and their polymers. ${ }^{31}$ GSE has exhibited promise as a vital distributor for the manufacture of new generations of antibacterial agents for dental use without exerting an influence on the biological equilibrium in the oral cavity. ${ }^{32}$ However, very limited research has been done in vivo. The process behind polyphenolic toxicity against microbes isdue to the suppression of hydrolytic enzymesor other interactions to inactive microbial adhesions and nonspecific interactions with carbohydrates. ${ }^{33}$ This infers the fact that phenolic antibacterial activity is due to enzymatic inhibition by the oxidized compounds which occurs through a reaction with sulfhydrylgroups with the proteins, ${ }^{33,34}$ additionally, flavonoids and tannins can bind to form precipitates with various proteins. ${ }^{35} \mathrm{~A}$ study by Aurelia et al. ${ }^{36}$ found that GSEs showa bacteriostatic effect on the anaerobic group that significantly diminishes the formation of biofilm. Dhillon et al. ${ }^{37}$ concluded that GSE has substantial antibacterial activity against mutans. Because various phytochemical studies of GSE exhibited antibacterial and antioxidative activities against S. mutans, we decided to formulate its mouthwash to check its efficacy and substantivity. Simultaneously, we wanted to know its comparison with other nutraceutical food products and chlorhexidine.

Chlorhexidine is considered to be thegold standard because of its antimicrobial property which is due to its di-cationic structure. Woodcock has reviewed the antibacterial action of bisguanides. ${ }^{38}$ The chlorhexidine group shows a wide range of susceptibilitiesto both Gram-positive and Gram-negative strains. As a mouthrinse, chlorhexidineshave numerous side effects.

Therefore, herbal mouthwashes using $P$. granatum, T. chebula, and Vitis vinifera were used in this study and a comparison was made among them in terms of $\mathrm{pH}$, buffering capacity, plaque index, and microbial assay. Chlorhexidine known to be the gold standard of all mouthwashes was kept as the control group.

On comparison of plaque deposition among all groups, it was found that $V$. vinifera had the lowest plaque deposition after 15 days followed by $T$. chebula and $P$. granatum. Increased antioxidant products like proanthocyanidins ${ }^{16}$ and other antibacterial properties of $V$. vinifera might have played a role in the lowest plaque deposition. After 30 days, the least increment of the plaque was noticed in P. granatum followed by groups T. chebula and $V$. vinifera. These results were similar to research done by Somu et al. ${ }^{39}$

The oral cavity maintains its $\mathrm{pH}$ at $6.2-7.6$ to make sure the effective maintenance of the integrity of tooth structure. Overall, the $\mathrm{pH}$ value obtained in the study was in the range of $7.0+3.0$ and 7.0-3.0. It showed that $\mathrm{pH}$ values were almost the same among all four groups at various time intervals which showed statistically nonsignificant results. Our study was in accordance with the study conducted by Pandey et al. ${ }^{40}$ which has shownthat no significant correlation was found between $\mathrm{pH}$ values and caries activity, age, and gender. Similarly, results produced by Carounanidy et al., ${ }^{23}$ Nayak et al., ${ }^{18}$ Vidhya et al., ${ }^{24}$ and Dilshad et al. ${ }^{41}$ were homogenous with our results.

The buffering capacity measured in all four groups wasalmost the same at the baseline day, days 16, and 30 that was statistically nonsignificant. The buffering capacity obtained was between 2.40 and 2.73 which is lower than the normal value. Our study included subjects which had a low DMFT score that might have resulted 
in low buffering capacity. According to Neil, the normal range of buffer capacity in saliva is $3-30 \mathrm{mg} / 100 \mathrm{~mL}$. Our results were in accordance with the result obtained by Carounanidy et al. ${ }^{23}$ and Garewal et al. ${ }^{42}$

The colonial growth of S. mutans in the saliva sample of P. granatum was found to be the lowest after 15 and 30 days. This was further followed by the $V$. vinifera group in 15 days category. Comparatively, $T$. chebula had shown less microbial growth than $V$. vinifera after 30 days. The presence of antioxidants and phytochemicals present in $P$. granatum, $V$. vinifera, and $T$. chebula might have reduced the $S$. mutans count in children. Results for $P$. granatum were in accordance with Prashanath et al. ${ }^{43}$ and Vahabi et al. ${ }^{44}$ The significant mutans' reduction was also found in Dilshad et al. ${ }^{41}$ Results for T. chebula were similar to Carounanidy et al., ${ }^{23}$ Nayak et al., ${ }^{45}$ Bajaj et al., ${ }^{46}$ and Garewal et al. ${ }^{42}$ Our result was dissimilar to the result obtained by Bansal et al. ${ }^{20}$ This can be attributed to facts like difference in formulation of mouthwash preparation, methodology, usage of mouthwash, etc.

This study has revealed the fact that nutraceutical food products produced better results than chlorhexidine. Vitis vinifera (group C) had produced the lowest plaque score after 15 days. Whereas P. granatum (group A) has shown a maximal reduction of S. mutans colony count both after 15 and 30 days. Punica granatum had the lowest plaque deposition after 15 days. Thus, it was observed that $P$. granatum has the ability to show maximal substantivity than others. Natural mouthwashes are not only efficacious but also have thepotential to be on common platform with chemical mouthwashes. Thus, undivided attention should be given to natural mouthwashes and oral health education.

The study was not without its restraints. Children mentioned about the bitter taste of the $V$. vinifera group. It had a small sample size in all groups, consequently reducing the power of the study, thereby increasing the margin of error. In addition, this study was self-funded. Further, the study can be performed on a large sample size with support from external sources.

The study decided to use chlorhexidine as the control group than keeping placebo in one of the groups as we wanted to compare nutraceuticals with the gold standard directly.

\section{Conclusion}

Thus, we can say $P$. granatum showed maximum substantivity. An increase in $\mathrm{pH}$ results in a decrease in caries activity which was seen in all groups. Subjects hadthe low DMFT score which resulted in low buffering capacity in all groups which infers that children were less prone to dental caries. All nutraceutical mouthwash can be safely used in children. This in vivo study implies that $V$. vinifera had shown the lowest plaque reduction owing to its antioxidant and phytochemical properties. Contrarily, $P$. granatum had shown better efficacy and substantivity. As it has been wisely said "Prevention is better than cure," we should emphasize more toward prevention of dental caries by using nutraceutical products which is a safe alternative and possess efficient quality.

\section{References}

1. Bhandari PR. Pomegranate (pumice granum L). Ancient seeds for modern cure? Review of potential therapeutic applications. Int J Nutr Pharmacol Neural Dis 2012;2:171-184.

2. Longtin R. The pomegranate: nature's power fruit? J Natl Cancer Inst 2003;95:346-348. DOI: 10.1093/jnci/95.5.346.
3. Fadavi A, Barzegar M, et al. Physicochemical composition of ten pomegranate cultivars (Punica granatum L.) grown in Iran. Food Sci Technol Int 2005;11:113-119. DOI: 10.1177/1082013205052765.

4. Aviram $M$, Dornfeld $L$, et al. Pomegranate juice consumption reduces oxidative stress, atherogenic modifications to LDL, and platelet aggregation: studies in humans and in atherosclerotic apolipoprotein E-deficient mice. Am J Clinl Nutr 2000;71:1062-1076. DOI: 10.1093/ ajcn/71.5.1062.

5. DiSilvestro RA, Silvestro DJ, et al. Pomegranate extract mouth rinsing effects on saliva measures relevant to gingivitis risk. Phytother Res 2009;23:1123-1127. DOI: 10.1002/ptr.2759.

6. Jagtap AG, Kerkira SG. Potential of the aqueous extract of Terminalia chaebol as an antiquary's agent. J Ethnopharmacol 1999;68:299-306. DOI: 10.1016/S0378-8741(99)00058-6.

7. Bag A, Bhattacharyya SK, et al. The development of Terminalia chebula Retz.(Combretaceae) in clinical research. Asian Pac J Trop Biomed 2013;3:244-252. DOI: 10.1016/S2221-1691(13)60059-3.

8. Williamson EM. Major herbs of Ayurveda. London: Churchill Livingstone; 2002. p. 299.

9. Juang LJ, Sheu SJ, et al. Determination of hydrolyzable tannins in the fruit of Terminalia chebula by highperformancc liquid chromatography and capillary electrophoresis. J Sep Sci 2004;27: 718-724. DOI: 10.1002/jssc.200401741.

10. Rangsriwong $P$, Rangkadilok N, et al. Subcritical water extraction of polyphenolic compounds from Terminalia chaebol Retz. Fruits Sep Purify Tech 2009;66:51-56. DOI: 10.1016/j.seppur.2008.11.023.

11. Thirumoorthyswamy S. Pleiotrophic Evaluation of Haritaki. Am J Phytomed Clin Ther 2014;2(1):33-44.

12. Kannan $P$, Ramadevi $S R$, et al. Antibacterial activity of Terminalia chebula fruit extract. Afr J Microbiol Res 2009;3(4):180-184.

13. Jayaprakasha GK, Negi PS, et al. Pomegranates: Ancient Roots to Modern Medicine. Boca Raton: CRC Press/Taylor and Francis; 2006; pp. 167-183.

14. Monagas M, Gómez-Cordovés C, et al. Monomeric, oligomeric, and polymeric flavan-3-ol composition of wines and grapes from Vitis vinifera L. Cv. Graciano, Tempranillo, and Cabernet Sauvignon. J Agric Food Chem 2003;51:6475-6481.

15. Baydar NG, Sagdic O, et al. Determination of antibacterial effects and total phenolic contents of grape (Vitis vinifera) seed extracts. Int J Food Sci Technol 2006;41:799-804.

16. Wu CD. Grape products and oral health. J Nutr 2009;139(9): 1818S-1823S. DOI: 10.3945/jn.109.107854.

17. Mandel ID. Chemotherapeutic agents for controlling plaque and gingivitis. J Clin Periodontol 1988;15(8):488-498. DOI: 10.1111/j.1600051X.1988.tb01020.x.

18. Nayak SS, Ankola AV, et al. Effectiveness of mouthrinse formulated from ethanol extract of Terminalia chaebol fruit on salivary Streptococcus mutans among 12 to 15 year old school children of Belgaum city: A randomized field trial. J Indian Soc Pedod Prevent Dent 2012;3(30):231-236. DOI: 10.4103/0970-4388.105016.

19. Anup N, Siddharth A, et al. Efficacy of commercially available herbal mouthrinses on the plaque and gingival status among 12-14 yrs old school children. Int J Bio Pharm Res 2015;6(3):233-235.

20. Bansal A, Marwah N, et al. Effect of Achyranthes aspera, $0.2 \%$ Aqueous Chlorhexidine Gluconate and Punica granatum Oral Rinse on the Levels of Salivary Streptococcus mutans in 8 to 12 Years Old Children. J Contemp Dent Pract 2015;16(11):903-909. DOI: 10.5005/ jp-journals-10024-1779.

21. Turesky S, Gilmore ND, et al. Reduced plaque formation by the chloromethyl analogue of victamine C. J Periodontol 1970;41:41-43. DOI: 10.1902/jop.1970.41.41.41.

22. Gupta D, Gupta R K, et al. Comparative evaluation of Terminalia chebula extract mouthwash and chlorhexidine mouthwash on plaque and gingival inflammation-4 week randomised control trial. Oral health Prev Dent 2015;13(1):5-12. DOI: 10.3290/j.ohpd.a32994.

23. Carounanidy U, Satyanarayanan $\mathrm{R}$, et al. Use of an aqueous extract of Terminalia chaebol as an anticaries agent: A clinical study. Indian J Dent Res 2007;18(4):152-156. DOI: 10.4103/0970-9290.35823. 
24. Rekha V, Jayamathi, et al. Anti cariogenic effect of Terminalia chebula. J ClinDiagn Res 2014;8(8):ZC51-ZC54.

25. PalomboEA. Traditional medicinal plant extracts and natural products with activity against oral bacteria: Potential application in the prevention and treatment of oral diseases. Evid Based Complement Alternat Med 2011;2011:680354. DOI: 10.1093/ecam/nep067.

26. Newman RA, Lansky EP, et al. Pomegranate: The Most Medicinal Fruit. Laguna Beach, California: Basic Health Publications; A Wealth of Phytochemicals; 2007; p. 120.

27. Endo EH, Cortez DA, et al. Potent antifungal activity of extracts and pure compound isolated from pomegranate peels and synergism with fluconazole against Candida albicans. Res Microbiol 2010;161:534-540. DOI: 10.1016/j.resmic.2010.05.002.

28. Varier. A dictionary of Indian raw materials and industrial products. New Delhi: Publications and Information Directorate, Council of Scientific and Industrial Research; 2002. pp. 387.

29. Kumar A, Lakshman K, et al. Estimation of rutin and quercetin Terminalia chebula by HPLC. Int J Aesth Antiag Med 2009;2(1):3.

30. Juang LJ, Sheu SJ, et al. Determination of hydrolyzable tannins in the fruit of Terminalia chebula by high-performancc liquid chromatography and capillary electrophoresis. J Sep Sci 2004;27(9):718-724. DOI: 10.1002/jssc.200401741.

31. Yoo MA, Chung HK, et al. Evaluation of physicochemical properties in different cultivar grape seed waste. Food Sci Biotechnol 2004;13:26-29.

32. Wu CD. Grape products and oral health. J Nutr 2009;139(9): 1818S-1823S. DOI: 10.3945/jn.109.107854.

33. Cowan MM. Plant products as antimicrobial agents. Clin Microbiol Rev 1999;12(4):564-582. DOI: 10.1128/CMR.12.4.564.

34. Song JH, Kim SK, et al. In vitro inhibitory effects of polygonumcuspidatum on bacterial viability and virulence factors of streptococcus mutans and streptococcus sobrinus. Arch Oral Biol 2006;51(12):1131-1140. DOI: 10.1016/j.archoralbio.2006.06.011.

35. Mehansho H, Butler LG, et al. Dietary tannins and salivary proline rich proteins: Interactions, induction, and defense mechanisms.
Annu Rev Nutr 1987;7:423-440. DOI: 10.1146/annurev.nu.07.070187. 002231.

36. Furiga A, Lonvaud-funel A, et al. In vitro study of anti oxidant capacity and antibacterial activity on oral anaerobes of a grape seed extract. JFoodchem 2009;113:1037-1040.

37. Dhillon J, Batra M. Antibacterial effect of grape seed extract against streptococcus mutans. APDJ 2015;2(1):9-12.

38. Woodcock PM. Biguanides as industrial biocides. In: Page KR. ed: Industrial biocides. Chichester: John Wiley; 1988. pp. 19-36.

39. Somu A, Ravindra A, et al. Efficacy of a herbal extract gel in the treatment of gingivitis: a clinical study. J Ayurveda Integr Med 2012;3(2):85-90. DOI: 10.4103/0975-9476.96525.

40. Pandey $\mathrm{P}$, Reddy VN, et al. Estimation of salivary flow rate, $\mathrm{pH}$, buffer capacity, calcium, total protein content and total antioxidant capacity in relation to dental caries severity, age and gender. Contemp Clin Dent 2015;6(Suppl 1):S65-S71. DOI: 10.4103/0976-237X.152943.

41. Umar D, Dilshad B, et al. The effect of pomegranate mouthrinse on Streptococcus mutanscount and salivary $\mathrm{pH}$ : An in vivo study. J Adv Pharm Technol Res 2017;7(1):13-16. DOI: 10.4103/2231-4040. 173266.

42. Garewal R, Garewal J, et al. Anticarious efficiency and effectiveness of Terminalia Chebula and Chlorhexidine as an oral rinse in children An In-Vivo Study. CODS J Dent 2015;7(1):8-12. DOI: 10.5005/cods7-1-8.

43. Prashanth DJ, Asha MK, et al. Antibacterial activity of Punica granatum. Fitoterapia 2001;72:171-173. DOI: 10.1016/S0367-326X(00)00270-7.

44. Vahabi S, Najafi E, et al. In vitro antimicrobial effects of some herbal essences against oral pathogens. J Med Plants Res 2011;5(19):4870-4878.

45. Nayak SS, Ashok Kumar BR, et al. The Efficacy of Terminalia chaebol Rinse on Streptococcus mutans Count in Saliva and Its Effect on Salivary pH. Oral Health Prev Dent 2010;8:55-58.

46. Bajaj N, Tandon S. The effect of Triphala and Chlorhexidine mouthwash on dental plaque, gingival inflammation, and microbial growth. Int J Ayurveda Res 2011;2:29-36. DOI: 10.4103/0974-7788.83188. 